happen at home and those reported to physicians at the time of the subsequent course.

Purpose To set up a comprehensive tool for AE reporting by patients and to assess whether it leads to an improvement in patients' quality of life.

Materials and Methods All consecutive patients treated in a day hospital oncological ward (digestive, thoracic, dermatological and haematology) over four courses of chemotherapy were included. A physician-patient logbook of 14 questions (rated from $1=$ absence to $4=$ strong) was completed daily during the first and third TIs. A global score was calculated for each course and compared to the results of QLQc30 forms.

Results Thirty-four patients were included, with a mean age of 59.9 and a male/female ratio of 1.3. A majority of metastatic diseases $(67.6 \%)$ had a WHO performance status (PS) score of $0 / 1$ $(88.2 \%)$. Most frequent AEs during the first TI were eating disorders $(1.72 \pm 0.11)$ and pain $(1.41 \pm 0.08)$. The daily score progressively decreased over subsequent TIs. Mean global score was $1.31 \pm 0.06$ and $1.14 \pm 0.06$ after the first and third TI, respectively. The frequency of all side effects decreased between the first and third courses. Eating disorders $(1.28 \pm 0.10)$ and neuropathy $(1.23 \pm 0.08)$ were the most frequent AEs in the third TI. Results of OLQc30 forms showed an improvement of the quality of life between the first and fourth courses. Most important improvements concerned nausea/vomiting (respectively score 22.1 to 8.3 ) and loss of appetite (score 31.4 to 21.2 ).

Conclusions A better awareness of AEs of anti-cancer drugs may improve their management. The use of a logbook could be helpful, as its interpretation may be related to an improvement in the quality of life.

No conflict of interest.

\section{OHP-007 AN OLD FRIEND FOR MINIMISING COST: DIRECT INTRAVENOUS ADMINISTRATION}

doi:10.1136/ejhpharm-2013-000276.381

'S Ibañez Garcia, ${ }^{2 A}$ Puebla Martin, ${ }^{2}$ Vázquez Rodriguez-Barbero, 'R Pérez Serrano, ${ }^{2}$ A Ambrós Checa, 'C Blázquez Romero, 'BM Muñoz Cejudo, 'A Martín Siguero, 'MT Franco Sereno, 'C Encinas Barrios. 'Hospital General Universitario de Ciudad Real, Pharmacy, CIUDAD REAL, Spain; ${ }^{2 H o s p i t a l ~ G e n e r a l ~ U n i v e r s i t a r i o ~ d e ~ C i u d a d ~ R e a l, ~}$ Intensive Care Unit, CIUDAD REAL, Spain

Background The increase in drug spending and the decrease in resources make it necessary to look for strategies for minimising costs.

Purpose To describe the strategy for administering highconsumption intravenous drugs (IVd) directly and estimating the associated resources saved in an Intensive Care Unit (ICU).

Materials and Methods We obtained a list of drugs whose consumption in the ICU was more than 1,000 units/year.

After a literature review, we selected those that could be safely administered via IVd but are usually administered in intermittent intravenous infusion. We prepared a table containing instructions for their reconstitution and administration.

For four weeks two nurses administered the medicines that had been prescribed and were included in the table via IVd, recording: drug, time spent in preparation \& administration and adverse reactions related to the route of administration.

\section{After collecting data}

We estimated the direct cost savings in fluids if all drugs consumed by the unit and included in the table had been administered by IVd during 2010.

We compared the time spent on the preparation and administration of drug doses used in routine practise versus time used to implement this strategy.
Results The ICU used more than 1,000 units/year of each of 39 intravenous drugs, of which 12 were included in the table: metoclopramide, colistimethate, hydrocortisone, phytonadione, pantoprazole, amoxicillin/clavulanic acid, dexamethasone, piperacillin/ tazobactam, furosemide, methylprednisolone, meropenem and ranitidine.

The nurses made 117 administrations via IVd (following the usual procedure) of these drugs. The average time was 6.5 minutes for preparation and administration of each dose and no adverse reactions were detected related to the route of administration.

We estimate the ICU can save 28,000€/year

Conclusions Direct IV administration can be safe and efficient.

The extension of a programme of this type throughout the Hospital could increase efficiency and rational use of medicines significantly.

No conflict of interest.

\section{OHP-008 ANALYSIS OF COSTS AND CONSUMPTION OF MEDICAL DEVICES FOR EXTRACORPOREAL PHOTOCHEMOTHERAPY IN SIENAÍS UNIVERSITY HOSPITAL (AOUS)}

doi:10.1136/ejhpharm-2013-000276.382

F Fiori, C Laudisio, E Cesqui, D Paoletti, D Iozzi, C Castellani, G Gallucci, G Sasso MT Bianco, A Catocci. Azienda Ospedaliera Universitaria Senese, Pharmacy, Siena, Italy

Background Extra-corporeal photochemotherapy (ECP) is a procedure that exposes mononuclear blood cells, obtained through centrifugation, to ultraviolet irradiation, in the presence of the DNA binding agents such as 8-methoxypsoralen (8-MOP). Two methods can be used:

ON-LINE, which consist of irradiation of cells through extracorporeal circulation (the only method used in AOUS until 2011).

OFF-LINE, which consist of leukapheresis of concentrated lymphomonocytotic cells, irradiation and subsequent reinfusion (this method was introduced in AOUS in 2012).

Purpose The objective of this study was to analyse the costs and consumption data of the Medical Device (MD) necessary for ECP in the period 2007-2011, and make a prediction of costs and consumptions in the light of the introduction of the new method.

Materials and Methods We analysed the costs and consumption data of the MD used in ECP in AOUS, extrapolating from the hospital's computer database. Then an estimate of consumption and costs over the period was calculated. The literature and technical specifications of the $\mathrm{MD}$ were also consulted to find for what purposes ECP is indicated.

Results ECP is mainly used for T-cell-mediated diseases such as organ transplant rejection, systemic sclerosis, bullous pemphigus, acute and chronic graft-versus-host-disease (GvHD). The period considered to have the highest consumption was in 2008, with 956 kits consumed (at a cost of $€ 756,099.96$ ) and 5 UV lamps $(€ 7,987.50)$. In subsequent years, there was a progressive decrease in materials consumed. The average consumption was 867 kits/year, with cost/year of $€ 767,178.82$. The cost of an off-line ECP kit is $€ 300.99$ and a leukapheresis kit is $€ 169$.4. The estimated annual cost of the product if using the off-line method would be $€ 409,922.21$, versus $€ 914,080.83$ using the on-line method.

Conclusions By using the off-line method and the prices of the new contract, AOUS would save $55 \%$ compared to the current cost of the on-line method, equal to $€ 504,158.62$. A further savings factor is the fact that the lamps for the ECP with the new contract are provided free of charge. This will allow better reallocation of financial resources.

No conflict of interest. 